\title{
HeilberufeSCIENCE-Sonderausgabe zum 9. Gesundheitspflege-Kongress
}
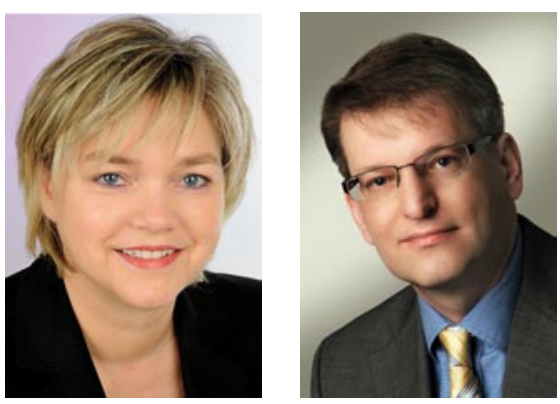

A. Tauchert

J. Klewer

Mit dieser Ausgabe erscheint die zweite HeilberufeSCIENCE-Sonderausgabe des Jahres 2011. Sie bietet Ihnen die Abstracts der Beiträge des 9. Gesundheitspflege-Kongresses, der am 25. und 26. November 2011 in Hamburg stattfand - einschließlich der Abstracts der Vorträge und Poster des HeilberufeSCIENCE-Symposiums. Alle Abstracts können Sie jederzeit einzeln von den HeilberufeSCIENCE-Seiten bei springerlink.de downloaden.

Die regelmäßigen Downloads der Inhalte früherer Sonderausgaben zeigen, dass sich die inhaltliche Verknüpfung von HeilberufeSCIENCE mit den Heilberufe-Kongressen erfolgreich etabliert hat und den Theorie-Praxis-Transfer verstärkt. Somit wird über das Online-Journal vielen Interessierten aus Forschung und Praxis der dauerhafte Zugang zu den Themen des 9. Gesundheitspflege-Kongresses erleichtert. Die publizierten Abstracts liegen in einer zitierfähigen Version für wissenschaftliche Arbeiten vor.

Die vorliegende Sonderausgabe gliedert sich in drei Teile:

Der erste Teil umfasst die alphabetisch gegliederten Abstracts ausgewählter Kongressbeiträge, welche die thematischen Schwerpunkte des 9. Gesundheitspflege-Kongresses widerspiegeln, wie z. B. Akademisierung der Weiterbildung,
Führung, Ältere Patienten in der Akutversorgung, Selbstbestimmungsrecht, Versorgung Chronisch Kranker und Pflege von Patientinnen mit Mammakarzinom.

Der zweite und dritte Teil umfassen jeweils die Abstracts der kongressbegleitenden Postersession sowie die Abstracts des HeilberufeSCIENCE-Symposiums. Dort wurden wissenschaftliche Arbeiten aus den Gebieten Pflegemanagement, Pflegepädagogik, Pflegewissenschaften, Gerontologie und Gesundheitswissenschaften/Public Health, unter Einbeziehung der stationären und ambulanten Pflege sowie der Altenhilfe und Altenpflege, präsentiert und diskutiert. Dabei lag der Schwerpunkt auf den Themengebieten stationäre Altenhilfe und Berufsaussichten von Studierenden in Pflege-Studiengängen.

Wir laden Sie ein, sich mit dieser HeilberufeSCIENCESonderausgabe über die Kongressthemen $\mathrm{zu}$ informieren oder beim Kongress Gehörtes noch einmal zu vertiefen. Natürlich würden wir uns freuen, wenn wir Sie auch auf dem Kongress Pflege 2012 am 27. und 28. Januar in Berlin begrüßen dürfen. Und denken Sie daran: mit dem gesamten Angebot von Heilberufe sammeln Sie wertvolle Punkte im Rahmen der Registrierung beruflich Pflegender.

Ihre

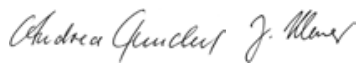

A. Tauchert - J. Klewer 